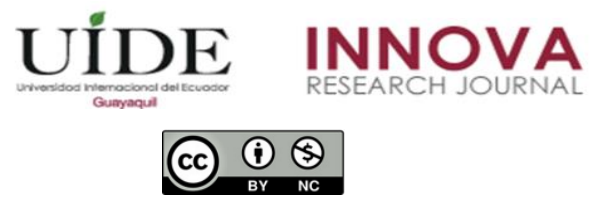

INNOVA Research Journal, ISSN 2477-9024

(Septiembre-Diciembre 2019). Vol. 4, No.3.2 pp. 188-204

DOI: https://doi.org/10.33890/innova.v4.n3.2.2019.1256

URL: http://revistas.uide.edu.ec/index.php/innova/index

Correo: innova@uide.edu.ec

\title{
Análisis de pertinencia en empresas con gestión del desempeño por competencias: Cantón Jipijapa
}

\section{Relevance analysis in companies with performance management by competencies: Jipijapa Canton}

Cesar Bustamante Chong

http://orcid.org/0000-0003-0150-1088

Universidad ECOTEC, Ecuador

Mariana Bustamante Chong

Universidad Estatal del Sur de Manabí, Ecuador

Michelle Varas Chiquito

Universidad de Guayaquil, Ecuador

Autor por correspondencia: cbustamante@ecotec.edu.ec; mariana.bustamante@ unesum.edu.ec; michelle.varasch@ug.edu.ec

Fecha de recepción: 15 de noviembre del 2019 - Fecha de aceptación: 13 de diciembre del 2019

\section{Resumen}

El siguiente artículo es de naturaleza teórica y tiene por objetivo presentar un análisis de pertinencia en empresas con gestión del desempeño por competencias, desde el punto de vista de la productividad, así como herramienta de desarrollo en el área urbana del cantón Jipijapa con el fin de mejorar factores internos y externos que afectan estas empresas. El progreso del movimiento económico, se manifiesta por la producción y mantenimiento de la eficacia del conocimiento para la estabilidad, la elaboración y el éxito de una organización, este entorno ha solidificado una atmosfera competitiva, en el que las empresas deben ofertar la innovación incesante de sus ventajas competitivas a través de permanentes renovaciones y el desarrollo de nuevos conocimientos y capacidades. La metodología que se utilizó es de origen mixto y se recurrió a tres de sus técnicas que son la observación, la revisión bibliográfica y de campo. Mejoramiento de la gestión del desempeño por competencias para dotar de personal idóneo a la empresa, innovación a través de la tecnología, la competencia del perfil profesional de sus empleados, determinación de los puestos laborales que ocupen o aspiren, de acuerdo a las necesidades de la empresa son los resultados esperados del constantemente cambio a consecuencia de la globalización para contribuir en la eficiencia de la empresa.

Palabras claves: competitividad; innovación; productividad; conocimientos; capacidades

\begin{abstract}
The following article is theoretical in nature and aims to present an analysis of relevance in companies with performance management by competencies, from the point of view of productivity, as well as development tool in the urban area of the Jipijapa canton in order to
\end{abstract}


improve internal and external factors that affect these companies. The progress of the economic movement is manifested by the production and maintenance of the efficiency of knowledge for the stability, development and success of an organization, this environment has solidified a competitive atmosphere, in which companies must offer the incessant innovation of its competitive advantages through permanent renovations and the development of new knowledge and skills. The methodology that was used is of mixed origin and three of its techniques were used, which are observation, literature review and field. Improvement of performance management by competencies to provide the company with suitable personnel, innovation through technology, the competence of the professional profile of its employees, determination of the jobs they occupy or aspire, according to the needs of the company. Company are the expected results of the constantly changing as a result of globalization to contribute to the efficiency of the company.

Key words: competitiveness; innovation; productivity; knowledge, skills

\section{Introducción}

Una de las principales maneras para que una empresa pueda lograr una economía competitiva proviene de la gestión del conocimiento desde la cultura y las competencias de los individuos. En cuanto a la gestión por competencias, (Navimipour, 2015 ) plantea que la gestión por competencias de los individuos y los colectivos, en el marco de la gestión de conocimiento, es el conjunto de procesos que determina la creación, distribución y utilización de habilidades y conocimientos de los recursos humanos, los cuales son el activo más importante de cualquier organización y el factor clave para su éxito y cumple con los objetivos mediante el uso de su fuerza, experiencia, conocimiento, educación y habilidades.

En este artículo se detalla primero un acercamiento a la situación objeto de estudio, todo esto con el fin de detallar la importancia que tiene la investigación en el cantón Jipijapa, el análisis de pertinencia en empresas con gestión del desempeño por competencias.

En la segunda parte todo lo concerniente a conceptos básicos y primordiales que permiten obtener los fundamentos y bases necesarias, que describe la importancia, objetivos, componentes y herramientas útiles para la comprensión y el desarrollo de un subsistema de talento humano basado en competencias para el reclutamiento, selección y contratación de personal, detallándose el diagnóstico situacional de dicho proceso en las empresas objeto de estudio, en el cual se utilizó, diferentes técnicas e instrumentos como son entrevista y encuesta dirigida tanto a los jefes departamentales como al resto de colaboradores, para recolectar información con el fin de determinar la situación actual de dicho proceso y las diferentes necesidades que tiene el departamento de recursos humanos para el desarrollo del mismo. En la tercera parte se detalla la metodología, resultados de las encuestas, análisis, discusión, y un análisis general de los resultados. Finalmente se plantean las conclusiones obtenidas del diagnóstico situacional del análisis de pertinencia en empresas con gestión del desempeño por competencias en el cantón Jipijapa.

El objetivo del presente trabajo es: analizar la pertinencia en empresas con gestión del desempeño por competencias, desde el punto de vista de la productividad, así como herramienta de desarrollo en el área urbana del cantón Jipijapa con el fin de mejorar factores internos y externos que afectan estas empresas. 


\section{Problemática}

En la actualidad, la competitividad en las organizaciones es alta. Es por esta razón que, deberá seleccionar personal altamente competente y con experiencia para ofrecer un servicio eficaz y eficiente a los consumidores internos y externos, contando con procedimientos que concuerden en los diferentes cambios que se presentan, de tal manera que contribuyan a la buena administración y funcionamiento de sus procesos, como también al logro de los objetivos institucionales.

El talento humano en la organización para que sea considerada apta, y que pueda ocupar un puesto de trabajo dentro de la misma debe poseer experiencia, conocimientos, habilidades y destrezas, obteniendo ventajas en el desarrollo de sus actividades eficientemente, siendo el área de recursos humanos de vital importancia para cubrir dicha necesidad.

Los negocios micro empresariales comerciales o de servicios, personal o familiar ubicadas en el cantón Jipijapa, presentan desactualización y desorganización de sus procedimientos, debido a que no cuentan con la documentación donde se implantaran las ocupaciones, paso a paso que debían realizar para contratar y capacitar a sus colaboradores, lo cual afecta la calidad del servicio.

Se lleva a cabo el diagnóstico en el departamento de talento humano de las diferentes empresas del cantón pertenecientes a la cámara de comercio, con la ayuda de los empleados de las áreas involucradas, proponiendo realizar un levantamiento de procedimientos, los cuales servirían para optimizar y actualizar la gestión del desempeño por competencias. Llevando un mayor control de las tareas a realizar de una manera óptima, para proveer de personal capacitado que brinde el servicio de calidad deseado por el cantón.

\section{Competencias}

Los conocimientos por competencias de las distintas teorías de motivación, investigan la explicación del desempeño laboral exitoso mediante la identificación de altos potenciales, que pueden perfeccionarse, formando al personal y así cierran brechas para organizar su perfil de acuerdo a los requerimientos de la organización. Existen diversos libros y estudios acerca de las competencias y su funcionalidad, sin embargo, en el libro "Selección de Personal: Enfoque Clásico y de competencias" escrito por Jaime Moreno se propone que el enfoque de competencias busca identificar a los individuos de alto rendimiento para determinar cuáles son sus características personales que los distinguen (Moreno, 2000). En las organizaciones se están implementando procesos por competencia con la finalidad de generar mayor productividad. Los procesos más ejecutados son los de selección de personal y evaluación del desempeño.

Según diversos autores pueden existir varios tipos de competencias, pero se tomó en cuenta los tipos de competencias que son utilizados con frecuencia en el mercado laboral que son las competencias universales y específicas. 
Las competencias universales son conocidas como competencias genéricas, transversales, cardinales institucionales. Hacen referencia a las competencias que están presentes en todos los colaboradores. Representan la esencia de la misión y visión de la organización (Garcia J. , 2016)

Y las competencias específicas conocidas también como competencias técnicas. Son aquellas que buscan ser desarrolladas en un individuo para desarrollar funciones propias de un cargo puntual (Simms, 2005)

\section{Importancia de la Gestión por Competencias}

De entre las primeras definiciones que se conocieron sobre el significado de gestión por competencias, era la que ponía énfasis en el logro de objetivos mediante las capacidades personales de cada uno de los trabajadores, su rasgo y sello distintivo, cualidades propias de las personas que imprimen un contraste en el entorno de trabajo.

Toda buena gestión implica una eficaz administración de personal; la gestión por competencias es el conjunto de políticas, normas, órganos e instrumentos que, aplicados racional y coordinadamente, permiten llevar a cabo los procesos de vinculación, desarrollo, remuneración, relaciones laborales y de bienestar de los empleados con el propósito de facilitar el rendimiento organizacional (Arrobo, 29 de noviembre de 2013)

Las organizaciones son fundamentalmente grupos de personas que practican diversas ocupaciones directamente interrelacionadas con la intención de lograr una meta. Debido a esto, el factor decisivo de una organización eficaz es el contingente humano con el que se trabaja.

La labor dentro del área de talento humano dentro de las organizaciones está relacionada directamente con potencializar el desarrollo de las personas como seres humanos, como trabajadores de una empresa y ponderar el beneficio de los intereses de la institución.

La evidente importancia de las competencias en la práctica profesional ha estimulado discusiones y acciones que se inscriben en el progreso actual de la nombrada gestión estratégica (por competencias) de los recursos humanos.

En consecuencia, la competencia profesional: es una cualidad humana que responde bajo la integración de diversos conocimientos: saber, saber hacer, y saber ser. Los cuales radican en el comportamiento del empleado y hacen evidente su desempeño laboral, que le permiten desenvolverse en un espacio profesional y humano situado al más alto nivel de exigencia que ofrece la organización en cargos relativos a la profesión.

En la competencia profesional se manifiestan, entre otros engranajes, valores que son propios del sujeto, manifestados mediante toda su carrera profesional, y pulidos en su último desenvolvimiento; que evidencia un desempeño eficaz, independiente, dúctil, variable, sostenible con miras a la inventiva personal.

Por lo tanto, el desempeño profesional se edifica en medida de la trascendencia desarrollada dentro de las competencias, las que cualifican y aseguran las exigencias requeridas 
dentro del ámbito socio laboral, en áreas donde desempeña las actividades, labores o roles ligados, más no forzadamente, a la realización de las funciones y procesos vinculados a la profesión.

Tejeda dentro de este aspecto sostiene: "El desempeño, es una actividad necesaria para el logro de los resultados exigidos en el ámbito profesional que supone diversos grados de complejidad..." (Tejeda, 2015)

Sin embargo el desarrollo de la competencia en las personas no es tan simple como parecería, hay que adentrarse a los comportamientos internos como ya se ha mencionado, en esta línea (Alles, 2006)afirma que "Cuando se trabaja con adultos como sucede en el ámbito de las organizaciones, la dificultad mayor está, frecuentemente, en que se debe lograr que las personas dejen de lado conocimientos obsoletos y los reemplacen por otros nuevos"

De allí que es muy importante identificar de manera clara, las verdaderas competencias que el cargo requiere, pues estás serán concluyentes a la hora de evaluar el desempeño de las personas en sus puestos de trabajo.

Los comportamientos son observables en cómo la persona ejerce su trabajo y las funciones determinantes del puesto, también en como procede y actúa con sus demás compañeros y consecuentemente en el proceso de su propio crecimiento profesional, lo cual dignifica a la empresa con actitudes positivas y compartiendo con el resto estas mismas actitudes para el logro de objetivos planteados.

Todos estos comportamientos deberán ser compartidos para que el compromiso grupal surja y sea sólido para el desarrollo, conjuntamente con la eficacia y la eficiencia las cuales van de la mano porque se debe tener eficacia en el cumplimiento de objetivos por parte del personal tomando en cuenta lo que debe hacerse para lograrlos con la eficiencia, predisposición y el uso preciso de los medios para llegar a las metas propuestas como lo menciona (Quintana, 2015) en su tesis sobre formación por competencias laborales, donde se observa conexión con el presente proyecto de titulación por tratarse del estudio de un tema en común, aplicado a una empresa distinta.

\section{Gestión de recursos humanos y sus subsistemas}

La gestión de recursos humanos es una predisposición que se está aplicando con mucho éxito en instituciones de vanguardia en diversos países a nivel mundial con la finalidad de incrementar los niveles de productividad y rendimiento organizacional mediante la potenciación del desempeño laboral de las personas.

Conviene mencionar que diversas publicaciones empresariales como Fortune, HR magazine entre otras, subrayan el hecho de que la gestión de recursos humanos por competencias es el enfoque que utilizan las organizaciones de alto rendimiento, como su nombre lo indica, estas organizaciones se caracterizan por una fuerte orientación a lograr al máximo desempeño en cualquier aspecto de su gestión. Es importante mencionar incluso que la gestión de recursos 
humanos por competencias, es el enfoque requerido por las normas ISO 9001:2000 para certificar a las organizaciones en función a la gestión de calidad.

Los subsistemas que administran recursos humanos en el siglo XXI son los siguientes:

- Subsistema de reclutamiento selección e inducción

- Subsistema de gestión del desempeño

- Subsistema de desarrollo organizacional y plan carrera

- Subsistema de administración de remuneraciones

- Subsistema de capacitación

- Subsistema de seguridad industrial y salud ocupacional

\section{La gestión del desempeño por competencias}

Una herramienta esencial para predecir el desempeño de un colaborador dentro de la organización es la gestión del desempeño por competencias, la misma que permite detectar habilidades y desarrollarlas (Wang, 2017)

Igualmente (Monir, 2015 )explican que una habilidad importante por el cual se debe caracterizar a un trabajador del conocimiento es la capacidad de comunicarse con los demás de una de manera constructiva.

El entorno organizacional se ha convertido en uno de los más importantes, ya que afecta directamente su desarrollo. Por lo tanto, es necesario establecer y analizar los diferentes factores y fuerzas que surgen fuera de sus fronteras, a las que están necesariamente sujetos, ya que afectan a su comportamiento. (Mora Pisco, 2016)

\section{Proceso de la gestión del desempeño por competencias}

Los sistemas de gestión del desempeño por competencias se describen como herramientas clave para transformar el talento y la motivación de las personas en una ventaja organizativa estratégica (Aguinis, Performance management. , 2014)

En el libro de Álvaro Herrera, (Herrera, 2014) recomienda que un proceso adecuado de la gestión del desempeño por competencias se basa en difundir adecuadamente las competencias de la organización, a su vez, de un cargo en específico. Estas competencias requeridas se las puede fortalecer desarrollándolas con capacitaciones o coaching, de tal forma que se cierren brechas. Formando al personal se evaluará las competencias que requieren para poder incrementar su índice de productividad, analizar los resultados e identificar oportunidades de mejora.

El proceso de gestión del desempeño por lo general se basa en dos ejes principales: los resultados y la adecuación del rol, el mismo que se basa en los requerimientos del cargo. Los resultados se basan en valorar los resultados en base a un periodo de evaluación (Aguinis, Performance management. , 2014)La gestión del desempeño no valora la persona en sí, sino su actuación en diversas situaciones buscando conocer el desempeño de la persona y su capacidad de resolver problemas que puedan surgir y a su vez, fomentar el desarrollo constante del 
personal. La finalidad de la gestión del desempeño es el tomar decisiones en cuanto al entrenamiento del personal, rotación de puestos, desarrollo de personal, planes de carrera y verificación del proceso de selección.

Existen autores de la gestión del desempeño por competencias que lo consideran como un proceso que permite alinear la labor diaria de cada persona con el cumplimiento de los objetivos y estrategias de la organización, y para ello se debe alinear las metas del desempeño individual a las metas vinculadas con la estrategia del negocio.

\section{Análisis del Sector y de la empresa} del Ecuador.

El cantón Jipijapa, está ubicado al sur de la provincia de Manabí. En la franja costera

Su superficie es de $1.420 \mathrm{Km}^{2}$, cuenta con 81598 habitantes con una población económicamente activa de 20.561 personas (Censo 2001INEC), representa el 5,5\% del total de la Provincia de Manabí; ha crecido en el último período intercensal 1990-2001, a un ritmo del 0,5\% promedio anual. El 45,2 \% de su población reside en el área rural; se caracteriza por ser una población joven, así el 42,7\% son menores de 20 años, según se puede observar en la Pirámide de Población por edades y sexo.

Está ubicado al sur de Manabí y es conocido también como "La sultana del café" por ser el principal productor y exportador de café en lo que basa la economía su población, además del comercio en sus diferentes formas y la artesanía. Entre sus recursos turísticos tiene las playas de Puerto Cayo.

La zona urbana del cantón Jipijapa se encuentra constituido por las parroquias de: Dr. Miguel Morán Lucio, Manuel Inocencio Parrales y Guale y San Lorenzo de Jipijapa, siendo ésta la más poblada. Entre las zonas de influencias de este sector están:

- Centro cuya calle principal es la Bolívar que cuenta con numerosos restaurantes, locales de comercio, servicios, licorerías y bares, almacenes Tía, Super despensa Aki.

- Los Ángeles arteria principal es la avenida universitaria donde se asientan numerosos restaurantes locales de comida rápida y típica centros de servicios y demás.

- Av. vía Puerto Cayo donde se encuentra ubicado el centro comercial mi comisariato, instituciones públicas y que cuenta con locales comerciales.

Mediante el presente artículo se define la estructura del sector micro empresarial tanto formal como informal de la zona urbana del cantón Jipijapa para así poder establecer las estrategias a seguir.

Tiene como propósito el análisis de pertinencia en empresas con gestión del desempeño por competencias: cantón Jipijapa, con lo cual se estaría contribuyendo a mejorar las constantes problemáticas que se presentan a nivel empresarial entre patronos y empleados por la ausencia de un departamento estructurado que se encargue de todo lo relacionado al manejo del personal con sus operaciones. 
Siendo la innovación un factor decisivo para la productividad de cualquier tipo de empresa, resulta necesario ante el escaso desarrollo que poseen las microempresas familiares de Jipijapa, se impulsen mecanismos de apoyo a las actividades innovadoras en conjunto con la Universidad estatal del sur de Manabí, los gobiernos autónomos descentralizados y gobierno central.

En el cantón Jipijapa, existen un gran número de microempresas, como una alternativa de escasez de oferta laboral, creando sus propias iniciativas como respuesta a esta escasez, como fuente de ingreso para su sustento, éstas enfrentan una problemática que se relaciona al escaso conocimiento para contratar personal idóneo, manejo de leyes reglamentarias.

El presente artículo se desarrolla en la zona urbana del cantón Jipijapa , provincia de Manabí, con las microempresas que se encuentran en el cantón Jipijapa, considerando que éstas, no cuentan con profesionales especializados en sus puestos de trabajo.

\section{Situación Actual}

Actualmente las empresas están en una constante competitividad, siendo motivo suficiente de demanda de recursos humanos eficientes. El ser humano en muchas ocasiones se encuentra afectado por variables como las capacidades, competencias y esquemas de comportamientos diversos, esto trae como consecuencia que las instituciones se vean obligadas a una investigación integra de excluir el mejor recurso humano para su buen trabajo o actividad asignada, siendo ésta la administración de Talento Humano.

Con insistencia se atribuye a la pequeña y mediana empresa una cierta concisión de retroceso hacia nuevos métodos y procedimientos de gestión de recursos humanos; herramientas que han evidenciado ser eficientes en las grandes organizaciones como contestación a los actuales requerimientos del entorno sociocultural, tecnológico y económico.

Antes de recriminar a las Pymes su escepticismo y tendencia crítica en materia de personal, se debe preguntar el porqué de esta actitud que, con sólo profundizar un poco, parecería más indicativa de salubridad organizativa que de carencia de visión directiva (Soto Pineda, 2004)

Las constantes variaciones originadas de la globalización intervienen claramente en la obtención diaria de las organizaciones, exigiéndose a establecer de algún modo extremo a estos cambios que participan diariamente, periódicamente en el rendimiento de trabajar de manera eficaz en lograr los objetivos que estos cambios conllevan, es aquí que se direcciona la táctica del recurso humano como capital humano, su eficacia reside en el proceso de sus contenidos y desarrollar sus capacidades como componente primordial que es el de valerse por sí mismo y entregar lo mejor a la organización.

\section{Talento Humano}


Según (CABRALES SALAZAR O. (.-C., 2009) la gerencia del talento humano bajo la perspectiva de la condición humana, revista Facultad de Ciencias Económicas: Investigación y Reflexión, Universidad Militar de la Nueva Granada-Colombia, vol. XVII, NUMERO 1, PAG $155-178$

Se ha considerado en el mundo laboral de hoy al Talento Humano como una de las variables más importantes en que las organizaciones deben centrar gran parte de su atención y sus recursos Como seres humanos cuya impronta debería el permanente crecimiento espiritual e intelectual, e inmersos en un contexto competitivo, se debe tomar conciencia de la particularidad de cada trabajador y de las competencias personales para desarrollarlas y potenciarlas. De la misma forma, si el gerente de hoy no se interesa por conocer a profundidad los intereses y expectativas de su equipo de trabajo, no podrá gestionar sus talentos y afinar sus intereses estratégicos de la organización con los de cada uno de los trabajadores.

Este servicio emprende ingresando en cambios interiores, innovación continua, concibiendo la realidad, misión, instrumento indispensable para plantear este reto es la gestión de competitividades, para enaltecer la calidad de perfección en el que hacer de la organización.

En materia de talento humano, aparece la calidad y disponibilidad del recurso humano: las competencias difíciles de encontrar en los niveles operario, técnico, administrativo/ profesional y gerencial/ ejecutivo; la falta de compromiso del recurso humano con la empresa, costos de la mano de obra calificada y la baja calificación del recurso humano (Rodríguez, 2003)

\section{Metodología}

La metodología que se usa en el presente trabajo es de origen mixto: revisión literaria bibliográfica, estadística, conceptual a través de trabajos relacionados al tema; consultas web, ejecutándose el estudio de interrelaciones con énfasis local y regional, está centrado en la recolección de información, el posterior proceso de presentación y análisis de los resultados obtenidos.

- Se realiza estudio descriptivo.

- Se utiliza el método cualitativo y cuantitativo para la investigación.

Se debe considerar además que a pesar de ser más generales los planteamientos cualitativos deben situarnos en tiempo y lugar (Hernández, 2010)Al plantear el problema hay que considerar que la investigación cualitativa es conducida principalmente en los ambientes naturales de los participantes; las variables no son controladas ni manipuladas, debido a que generalmente son conceptos generales como emociones y vivencias.

Cuando se habla de elementos cuantitativos, se refiere a los datos que se obtienen mediante los análisis empíricos que se desarrollan en el proceso de construcción del conocimiento. En las investigaciones pedagógicas estos análisis están presentes en varios momentos del proceso: (Pérez O, Esquema conceptual, referencial y operativo sobre los modelos estadísticos en las investigaciones educativas. , 2007.) 
Se caracteriza por su forma cíclica y circular, frente a la posición lineal que adopta el análisis de datos cuantitativos.

\section{Investigación Primaria}

\section{Segmento:}

El segmento, se centra en negocios micro empresarial.

\section{Encuestas:}

- Dueños de negocios micro empresariales formales en marcha. Estos datos serán recopilados en la cámara de comercio de la ciudad de Jipijapa, mediante muestreo aleatorio se recogerán las aspirantes.

- Pymes informales, donde mediante observación, se visitarán locales que evidencien una actividad micro empresarial.

\section{Trabajo de Campo}

Las encuestas serán realizadas en los mismos lugares de trabajo de las microempresas.

\section{Población y muestra}

El presente artículo está dirigido hacia los negocios micro empresariales de diversas índoles sean comercial o de servicio ubicadas en el la zona urbana de la ciudad de Jipijapa, a través de dichos negocios se recabará información como fuentes directas sobre la problemática existente. De acuerdo a la definición de la microempresa establecida para el presente estudio, los negocios que entrarán a formar parte de este estudio serán las que tengan las siguientes características:

- Negocio personal o familiar

- Número de empleados de 1 a 70.

- Ubicados en la zona urbana del cantón Jipijapa.

Se considera como unidad de estudio a quien figure como el administrador de dicho negocio, sea éste el dueño o no del mismo.

\section{Cálculo de la muestra}

Para el presente estudio, se escogerá el muestreo estratificado, pues se dividirá a la ciudad de Jipijapa en cinco sectores:

\section{Norte}


2. Sur

3. Centro

4. Este

5. Oeste

La división corresponde a considerar que, en cada sector de la ciudad, existen agrupaciones diferentes de microempresas y dado que cada sector responde geográficamente y demográficamente a diferencias particulares, se pretende obtener una identificación de cada sector de la ciudad de Jipijapa, en exclusividad de la zona urbana ya que es ahí donde se

promoverán las actividades investigativas. Para ello $n_{0}=\left(\frac{Z \alpha / 2}{\varepsilon}\right)^{2} p q \quad n=\frac{n_{0}}{1+\frac{n_{0}}{N}}$ utilización de la fórmula expresión:

Donde:

$n_{0}$ : Cantidad teórica de elementos de la muestra.

n: Cantidad real de elementos de la muestra a partir de la población asumida o de los estratos asumidos en la población.

$N$ : Número total de elementos que conforman la población, o número de estratos totales de la población.

$Z \alpha / 2$ : Valor estandarizado en función del grado de confianza (1-a) $100 \%$ de la muestra calculada, que será del $95 \%$ en este caso el valor $Z \alpha / 2$ es igual a 1.96

$\varepsilon$ : Error asumido en el cálculo, en este estudio se ha trabajado con un error del $6 \%$

$q$ : Probabilidad de la población que no presenta las características: 0.5

$p$ : Probabilidad de la población que presenta las características. $p=1-q=0.5$

$$
\begin{aligned}
n_{0}=\left(\frac{1.96}{0.06}\right)^{2} 0.50(0.50) & =254.78 \\
n & =\frac{254.78}{1+\frac{254.78}{81598}}=250
\end{aligned}
$$

Para la asignación del tamaño muestral a cada sector se utilizó la separación uniforme que da la misma categoría a cada estrato en cuanto a tamaño de la muestra se refiere.

Se usa herramienta tecnológica Excel para tabulación de datos.

\section{Resultado de la encuesta}

\section{Análisis de Datos}




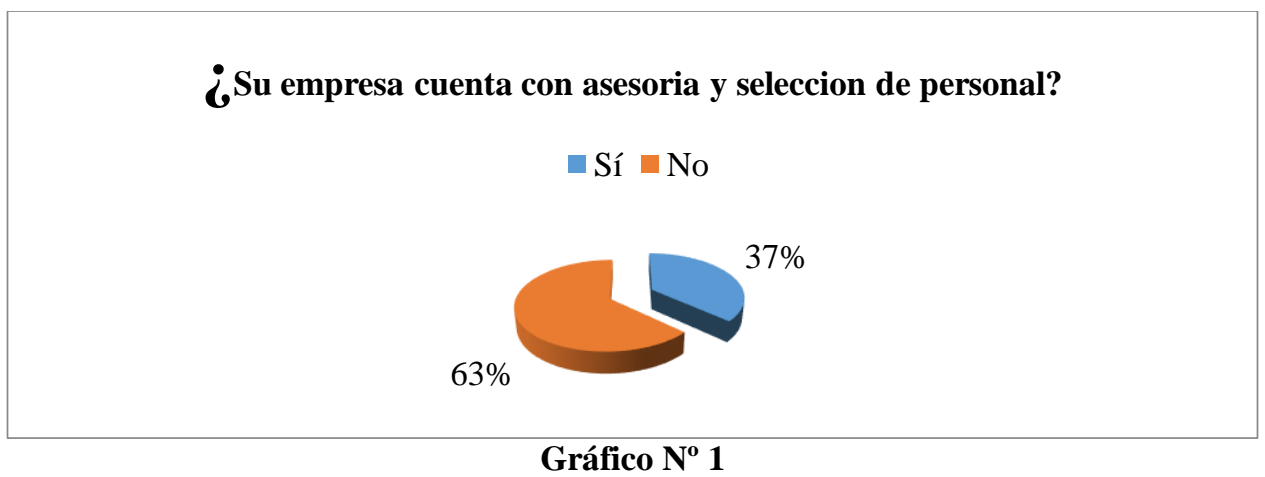

Análisis: De los encuestados el 63\% refieren que las empresas no cuentan con asesorías y selección de personal.

Comentario. Hay un gran porcentaje de brindar asesorías en las empresas del norte de la ciudad de Jipijapa.

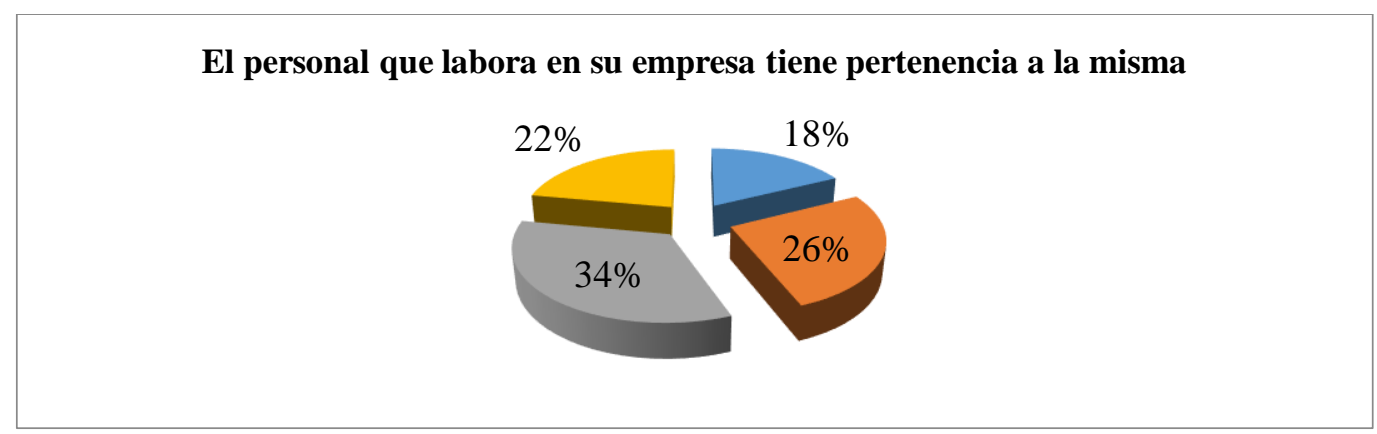

\section{Gráfico $\mathbf{N}^{\circ} 2$}

Análisis: De los encuestados si bien es cierto el $34 \%$ tiene pertenencia a la empresa, sin embargo, el $26 \%$ que es un buen porcentaje contribuye a que no estén consolidados, por tanto, afecta a la empatía y productividad de la empresa.

Comentario. Esto significa que, en el sector norte de la ciudad de Jipijapa, necesitaría contratar personal con más énfasis en la pertenencia.

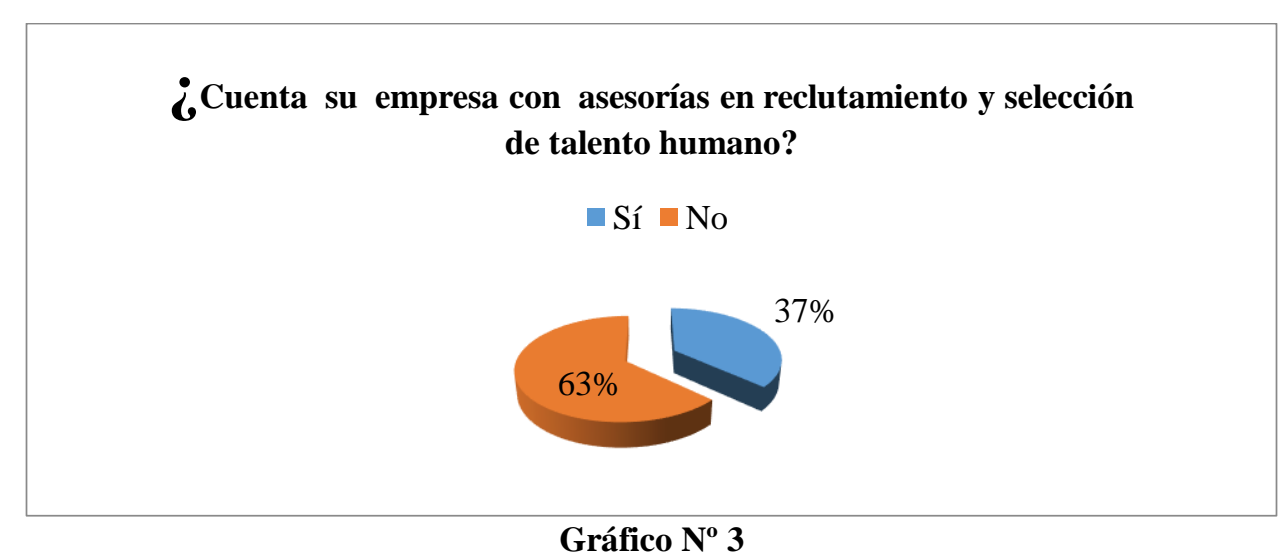


Análisis: De los encuestados el 63\% refieren que no cuentan con una empresa de asesorías en reclutamiento y selección de talento humano.

Comentario. Por lo que esto significa que hay poca asesoría en las empresas con la selección de personal y podrían optar por renovar sus recursos humanos.

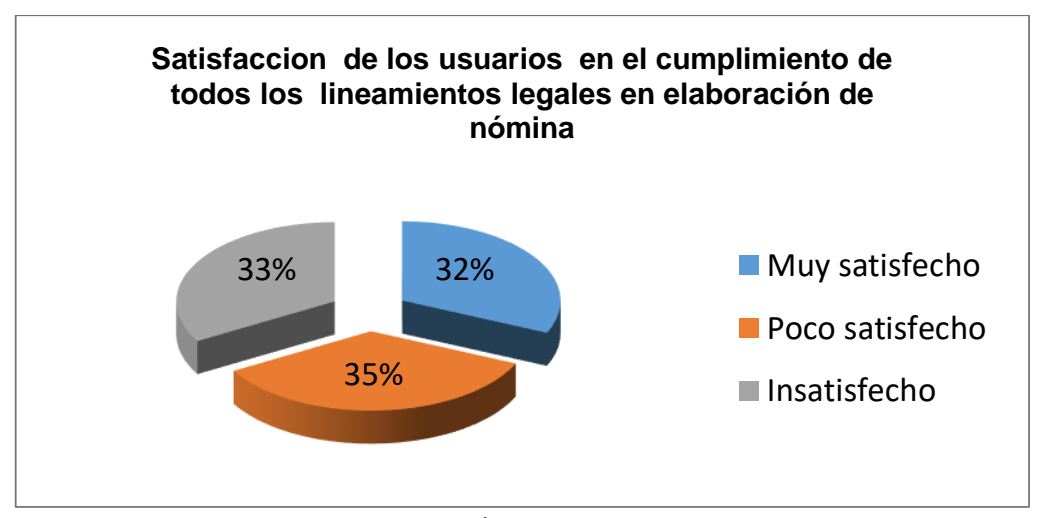

Gráfico $\mathbf{N}^{\circ} 4$

Análisis: De los encuestados el 32\% está muy satisfecho, el mayor porcentaje 35\% insatisfecho, con la elaboración de nómina que cumpla con todos los lineamientos legales.

Comentario. Esto significa que las pymes tienen que contratar personal idóneo para elaboración de nóminas.

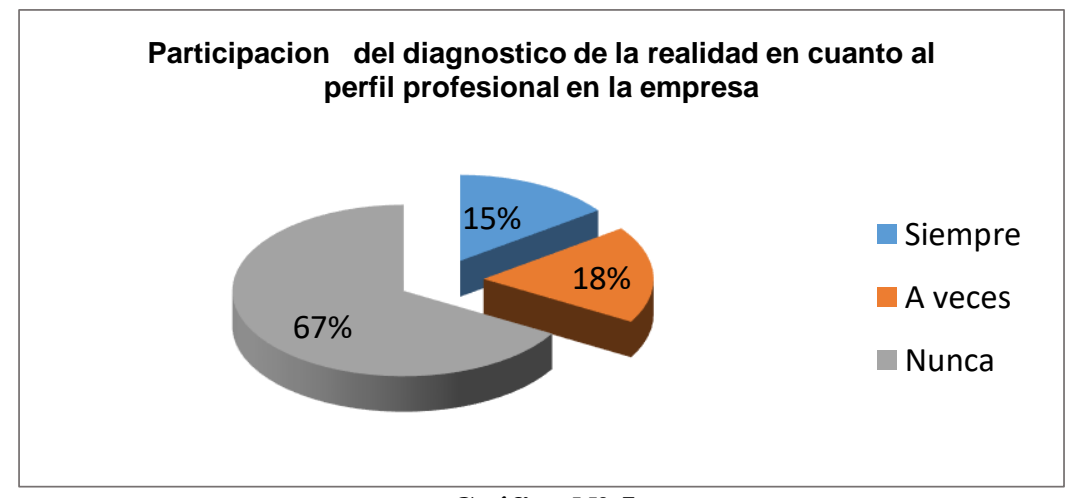

Gráfico $\mathbf{N}^{\circ} 5$

Análisis: El 67\% manifiesta que nunca se le ha participado del diagnóstico de la realidad en la empresa del perfil profesional.

Comentario. Existe gran posibilidad de aplicación de pertinencia en empresas con gestión del desempeño por competencias en el cantón. 


\section{Análisis general}

De las preguntas realizadas a los dueños de negocios micro empresariales formales en marcha, y las Pymes informales, se pudo verificar que un gran porcentaje de los microempresarios no cuentan con el personal altamente capacitado para realizar la tarea de seleccionar y elaborar nómina y en muchos casos son ellos mismos quienes deben realizar esta tarea descuidando sus principales funciones lo que como consecuencia da que sus negocios sean muy poco competitivos dentro de sus respectivos mercados. Por tanto, se considera que el presente trabajo investigativo si tendría un porcentaje alto de aceptación en el mercado basándose en el análisis de pertinencia en empresas con gestión del desempeño por competencias en el cantón Jipijapa.

\section{Conclusión}

Existe un componente básico que aceptan conocer los dueños de negocios micro empresariales formales en marcha, y las Pymes informales y sus inexactitudes, se insiste que es una herramienta ventajosa en donde se puede estar al tanto de diferentes aspectos y escoger información del mercado, además se logra instaurar las carencias de las empresas en cuanto a selección, reclutamiento y elaboración de nóminas y las habilidades que verifican.

Una vez comprobada la definición de los datos se puede concluir lo siguiente: la globalización de la economía y la emprendedora evolución del entorno exige a las organizaciones a una redefinición permanente y eficiente de las operaciones empresariales, en especial la de las Pymes.

La capacidad y el contexto financiero de las empresas objeto de estudio son aspectos importantes, que pueden incurrir en la presencia o ausencia de los espacios de recursos humanos dentro de su distribución; las empresas que por lo general no sobrepasan los 50 empleados, no tienen dicha área y las situaciones derivadas de la gestión de los individuos es obtenida por los dueños, gerentes u otro tipo de cargos de la empresa. Se expone que sólo las empresas medianas, donde la representación de empleados supera los cincuenta, reconocen personas representadas de gestionar el talento humano.

Un hallazgo importante de este artículo fue el hecho de localizar un número considerable de empresas que prescinden de las características demográficas de sus empleados, en aspectos relacionados con la edad, estado civil, formación académica y experiencia. Este entorno lleva a recapacitar que la administración de la información relacionada con el factor humano no es selectiva para las micro y pequeñas empresas, fundamentalmente teniendo en cuenta que estos datos aprueban direccionar programas de bienestar o mejoramiento de la calidad de vida de los trabajadores, contar con una base de datos del personal para proyección de necesidades, entre otros.

El carácter familiar que tiene este tipo de empresas hace que el reclutamiento y la selección se lleven a cabo de manera informal, por cuanto se basan en personas recomendadas por los mismos empleados para iniciar procesos de selección, y sólo realizan entrevistas, verificación de referencias y pruebas básicas de conocimiento del oficio para la contratación. 
En la época de la información, donde el conocimiento, la tecnología, la innovación y la creatividad es situación esencial de competitividad empresarial, la capacitación es uno de los métodos de gestión humana más descuidados, las pymes no cuentan con un análisis de necesidades, plan establecido previamente elaborado y no tienen presupuesto para desarrollar los programas. Lo que usualmente instruyen hacer es que de acuerdo a los requerimientos de la empresa se capacitan los interesados.

La evaluación del desempeño también es un proceso que se emplea de manera informal, pues se basa en los esquemas de producción por hora, sin llevar apropiadamente enfocados las informaciones. Lo que alarma respecto a dicho proceso es que no es un instrumento utilizado para el crecimiento y desarrollo de las personas, sino que es vista como un mecanismo para determinar la estabilidad del individuo en la organización y la posibilidad de otorgar la bonificación por resultados.

Los procesos de reclutamiento, selección, socialización, entrenamiento, capacitación y evaluación del desempeño se aplican de manera informal, cuando son fundamentales en el marco de la responsabilidad social interna, por cuanto ellos legalizan de ser aplicados a cabalidad un excelente ambiente de trabajo y una mejor calidad de vida laboral, una mayor responsabilidad, desarrollo de los trabajadores y un desempeño integral de la empresa.

Existe una relación directa asociada a la promoción y apropiación de la gestión de desempeño, relacionada con una cultura organizacional. En este sentido si se perfecciona en la adquisición y apropiación de competencias en los individuos, esta capacidad impulsara a la cultura organizativa, originando estrategias y propuestas de valor.

Entre las particularidades de la gestión de desempeño en las Pymes, tal como competencias para la gestión de la información y documentación, las competencias para la comunicación, las competencias para la innovación y el cambio, las competencias en diseñar herramientas digitales y las competencias para el aprendizaje organizacional, se relacionan con el talento humano adecuado, logrando reubicar a su entorno métodos, técnicas y tecnologías que apoyen a una excelente relación con los clientes internos y externos desde el orden local, regional y nacional.

Dada la importancia de la gestión del desempeño en el mundo empresarial actual, es concluyente evaluar el estado de su promoción en las empresas, pero también establecer, planes programas y proyectos que permitan llegar al estado ideal.

Finalmente, el éxito del análisis de pertinencia en empresas con gestión del desempeño por competencias en el cantón Jipijapa dependerá de diversos factores, no simplemente de uno en definitiva, por lo que llevar a cabo una calificada planificación, cronograma ajustado, personal calificado, supervisión de las actividades, depuración gradual de los datos y responsabilidad al trabajo son algunas de las variables que intervienen en el proceso, gestionadas de forma intelectual y metodológicamente. 


\section{Bibliografía}

Aguinis, H. (2014). Performance management. . Pearson. .

Aguinis, H. (2014). Performance management. . Pearson. .

Alles, M. (2006). Desarrollo del Talento Humano Basado en Competencias. Buenos Aires. Ediciones Garnica. .

Arrobo, W. J. (29 de noviembre de 2013). Modelo De Gestión Del Talento Humano Por Competencias de la empresa cimpexa s.a. tesis de grado previo a la obtención del título de ingeniero en administración de empresas de servicios y recursos humanos. guayaquil ecuador. universidad tecnológica equinoccial. .

Cabrales Salazar, O. (.-C. (2009). "La Gerencia Del Talento Humano Bajo La Perspectiva De La Condicion Humana”, Cabrales Salazar, Omar (2009), "La Gerencia Del Talento Humano Bajo La Perspectiva De La Condicion Humana. Nueva Granada-Colombia, vol. XVII, NUMERO 1, PAG 155-178: Revista Universidad Militar de la Nueva Granada-Colombia-

Escobar, F. R. (19 de Diciembre de 2007. Recuperado el 12 de ferbrero de 2015, de Gestión de Talento Humano). Gestión del Talento Humano. . : http://aprendeenlinea.udea.edu.co/lms/moodle/course/view.php?id=505.

Garcia, J. (2016). Desarrollo de competencias para el siglo XXI. . Humbolt International University Brochure. .

Hernández, R. F. (2010). Metodología de la Investigación, (5a ed.). . .

Herrera, Á. (2014). Géstion basada en competencias. Manpower Journey.López Gumucio, J. ricardo la selección de personal basada en competencias y su relación con la eficacia organizacional perspectivas . Universidad Católica Boliviana San Pablo Cochabamba, Bolivia, núm. 26, julio-diciembre, 2010, pp. 129- 152 .

Monir, H. y. (2015 ). The Relationship between Transformational Management and Communication Skills among Heads of Departments of KAU, . Procedia Computer Science, (65), 1160 - 1164.

Mora Pisco, L. L. (2016). Consideraciones actuales sobre gestión empresarial. . Obtenido de https://dialnet.unirioja.es/descarga/articulo/5802891.pdf , Mora Pisco, L. L., Duran Vasco, M. E., \& Zambrano Loor, J. G. (2016). Consideraciones actuales sobre .

Moreno, J. ( 2000). Selección de Personal: Enfoque Clásico y de Competencias. .

Navimipour, N. R. (2015 ). , Expert Cloud: A Cloud-based framework to share the knowledge and skills of human resources. Computers in Human Behavior, (46), 57-74 .

Pérez O, H. R. ( 2007.). Esquema conceptual, referencial y operativo sobre los modelos estadísticos en las investigaciones educativas. . Curso 87 Pedagogía 2007. La Habana Cuba. 2007.

Pérez O, H. R. ( 2007.). Esquema conceptual, referencial y operativo sobre los modelos estadísticos en las investigaciones educativas. . Curso 87 Pedagogía 2007. La Habana Cuba. .

Quintana, V. A. ( 2015). Diseño de un Sistema de Formación por Competencias Laborales en una empresa Productora. Ambato, Tungurahua, Ecuador. .

Rodríguez, A. (2003). La realidad de la Pyme colombiana. Desafío para el desarrollo. Colombia: Fundes.

Simms, H. (2005). Human Resource Planning. En S. Helen, HRM and competitive advantage . California: Select Knowledge, pág. 340.

Esta obra se comparte bajo la licencia Creative Common Atribución-No Comercial 4.0 International (CC BY-NC 4.0) 
Soto Pineda, E. \&. (2004). Las Pymes ante el desafío del siglo XXI: Los nuevos mercados globales. Thomson Learning.

Tejeda, R. (2015). Las competencias y su relación con el desempeño y la idoneidad profesional. . Revista Iberoamericana de educación, 9-12. .

Wang, S. \&.-C. (2017). Overcoming Human Capital Voids in Underdeveloped Countries. . Global Strategy Journal, 7(1), 36-57. http://dx.doi.org/10.1002/gsj.1144 . 